\title{
Virtual postoperative visits following robotic gynecologic surgery: a study of patient satisfaction, safety, and feasibility
}

\author{
Christina Mezes ${ }^{1}$. Jordan S. Klebanoff ${ }^{1}\left(\mathbb{D} \cdot\right.$ Ekaterina Grebenyuk $^{1}$. Joseph Gobern ${ }^{1} \cdot$ Sam W. Meske ${ }^{1}$. \\ Richard Amdur ${ }^{2}$. Gaby N. Moawad ${ }^{3,4}$
}

Received: 23 November 2021 / Accepted: 5 December 2021 / Published online: 31 January 2022

(c) The Author(s), under exclusive licence to Springer-Verlag London Ltd., part of Springer Nature 2021

\begin{abstract}
Since the onset of the COVID-19 pandemic the use of telehealth has burgeoned. Numerous surgical specialties have already adopted the use of virtual postoperative visits, but there is data lacking in both robotics and gynecology. In this single-institution prospective cohort study we sought to evaluate the patient satisfaction, feasibility and safety of postoperative telehealth visits following robotic gynecologic surgery. Thirty-three patients undergoing robotic gynecologic procedures participated in a postoperative telehealth visit approximately 2 weeks following surgery, of which 27 completed a survey which assessed participant satisfaction with the telehealth visit, overall health-related quality of life following surgery, exposure to telehealth visits, and social determinants of health. The mean satisfaction score was just below 'excellent'. Only 2 participants $(6.3 \%)$ required an in-person visit. Postoperative telehealth visit satisfaction score was significantly associated only with BMI (Pearson $r=0.45, p=0.018$ ). These data suggest that telehealth visits following robotic gynecologic procedures appear to be safe and feasible, and are associated with a high level of patient satisfaction.
\end{abstract}

Keywords Robotic surgery $\cdot$ Gynecology $\cdot$ Telehealth $\cdot$ Telemedicine $\cdot$ Postoperative

\section{Introduction}

The COVID-19 pandemic has reshaped U.S. healthcare delivery. Due to social distancing regulations, healthcare providers have adopted widespread use of telehealth visits [1]. Surgical specialties have already demonstrated successful implementation of telehealth visits into the spectrum of their care $[2,3]$. These data highlight the feasibility and patient satisfaction with virtual visits during a pandemic.

Jordan S. Klebanoff

KlebanoffJ@mlhs.org

1 Department of Obstetrics and Gynecology, Main Line Health System, 100 East Lancaster Avenue, Suite 301, Wynnewood, PA 19096, USA

2 Department of Surgery, The George Washington University Hospital, Washington, DC 20037, USA

3 Department of Obstetrics and Gynecology, The George Washington University Hospital, Washington, DC 20037, USA

4 The Center for Endometriosis and Advanced Pelvic Surgery, McLean, VA 22101, USA
Within the specialty of Obstetrics and Gynecology, telehealth visits have been implemented in a variety of patient encounters including well-woman visits, surgical consultation, preconception counseling, family planning, prenatal care, and abortion care [4-7]. Despite these data, no studies have evaluated telehealth in the postoperative setting. Data borrowed from other surgical specialties demonstrate that telehealth visits following surgery can be safely offered $[8$, 9], are associated with a high degree of safety, similar rates of missed appointments as in-person visits, and high levels of patient satisfaction [10,11].

A scarcity of data exists demonstrating the safety of postoperative telehealth visits for a gynecologic patient population. Theoretical concerns may include an increased incidence of postoperative complications, or at least a delay in diagnosis and intervention. Providers may also be concerned that patients will not be satisfied with their surgical care without in-person visits following surgery. These issues have not been demonstrated by other surgical specialties already implementing postoperative telehealth visits, and we should not further delay this overdue application of telehealth in our clinical practice. The aim of this study is to demonstrate the patient satisfaction, safety, and feasibility of postoperative 
telehealth visits following minimally invasive gynecologic surgery.

\section{Methods}

We performed a prospective cohort study at a single academic-affiliated community hospital between January 2021 and July 2021. Study approval was obtained by the local institutional review board (IRB\# E-20-5088). Eligible study participants included patients over the age of 18 scheduled to undergo a robotic gynecologic procedure by 2 minimally invasive gynecologic surgeons. Eligible procedures included hysterectomy, myomectomy, excision of endometriosis, diagnostic laparoscopy, or adnexal surgery. Only patients that spoke English as a primary language could enroll in the study. Patient characteristics were obtained through the electronic health record. Patients were excluded if they were deemed incapable of making medical decisions, were scheduled to undergo an abdominal or hysteroscopic procedure, or spoke a primary language other than English.

\section{Study procedure}

Patients were initially screened and consented at their preoperative visit for study participation which routinely occurred 1 to 2 weeks prior to the planned surgical procedure. Participants who agreed to enroll in the study were then scheduled for a postoperative telehealth visit approximately 2 weeks following their surgical procedure. During the study period, telehealth visits were conducted using an online video conferencing software, Zoom (Zoom Video Communications, Inc., San Jose, CA) with both audio and visual capabilities. Patient consent was obtained before any telehealth visit.

Study participants agreed to complete a single survey within $48 \mathrm{~h}$ following their postoperative telehealth visit. Each study participant was required to provide a personal email address to both facilitate the telehealth visit and distribute the post-visit survey. Participants were told at enrollment that the estimated duration to complete this survey would take less than $5 \mathrm{~min}$. The survey was 4 pages long and each page contained 1,5 , or 10 items, for a total of 21 items. There was no completeness check and participants were able to change their answers if they chose. Adaptive questioning was not used. Participants were unable to submit the survey more than once. Only completed surveys were analyzed. This survey was developed using Qualtrics (Qualtrics XM, Provo, UT) and response data was collected and stored on this platform. The post-visit survey was developed to determine participant satisfaction with the telehealth visit, overall health-related quality of life following surgery, exposure to telehealth visits, and social determinants of health. A previously validated survey assessing patient satisfaction with telehealth visits was adapted to fit this specific postoperative patient population [12] (Supplemental Figs. $1 \& 2$ ). To determine health-related quality of life following surgery, a previously validated survey, EQ-5D-3L, was used [13]. The survey questions used to identify social determinants of health and experience with telehealth visits were developed specifically for this study by the author group. Survey questions were not randomized. If study participants failed to complete the postoperative survey within $48 \mathrm{~h}$ of the telehealth visit they were sent 3 autogenerated reminders via the Qualtrics platform. If despite these reminders, the survey remained unanswered, the participants were considered lost to follow-up. No incentives were offered for completing the postoperative survey.

\section{Statistical analysis}

Assuming a mean satisfaction score of 30 with a SD of 5, we needed to enroll 30 participants in this study for a range for the $95 \% \mathrm{CI}<4$. Assuming a $10 \%$ loss to follow-up, we aimed to enroll 33 patients. Associations between categorical and continuous variables were analyzed using analysis of variance (e.g. travel time $\times$ satisfaction scale score). Associations between 2 continuous variables were analyzed with Pearson $r$ (e.g. age $\times$ satisfaction). Since most patients had low estimated blood loss (EBL), EBL was coded as $<100 \mathrm{~mL}$ or $\geq 100 \mathrm{~mL}$. Each of the binary health-related quality of life items was coded as $0 / 1$ to represent no/yes, and their scores were summed to create a single Functioning score. We recoded the 10 satisfaction items so that excellent $=1$, good $=2$, fair $=3$, and examined their inter-correlations with Pearson $r$, and the Cronbach's alpha, which is an index of internal consistency reliability. When $\alpha>0.80$, this indicates that the items may form a unidimensional scale. In that case, we computed the mean of item scores to represent the satisfaction scale score. This scale score has better reliability than any of the individual satisfaction items, and thus is preferable if the items are highly correlated. SAS (version 9.4, Cary, NC) was used for data analysis with $p<0.05$ being considered significant.

\section{Results}

There were 33 participants enrolled in this study, 27 of which had available satisfaction scores for an $82 \%$ response rate (Fig. 1). Of the 6 participants that did not complete the post-visit survey, 1 patient did not have the opportunity for a postoperative telehealth visit due to complications related to the surgery, and the other 5 were lost to follow-up. Demographic data for the sample can be found in Table 1 . Mean age was $42 \pm 10$ years, mean Body Mass Index (BMI) was $33 \pm 9 \mathrm{~kg} / \mathrm{m}^{2}$, and the mean health-related quality of 


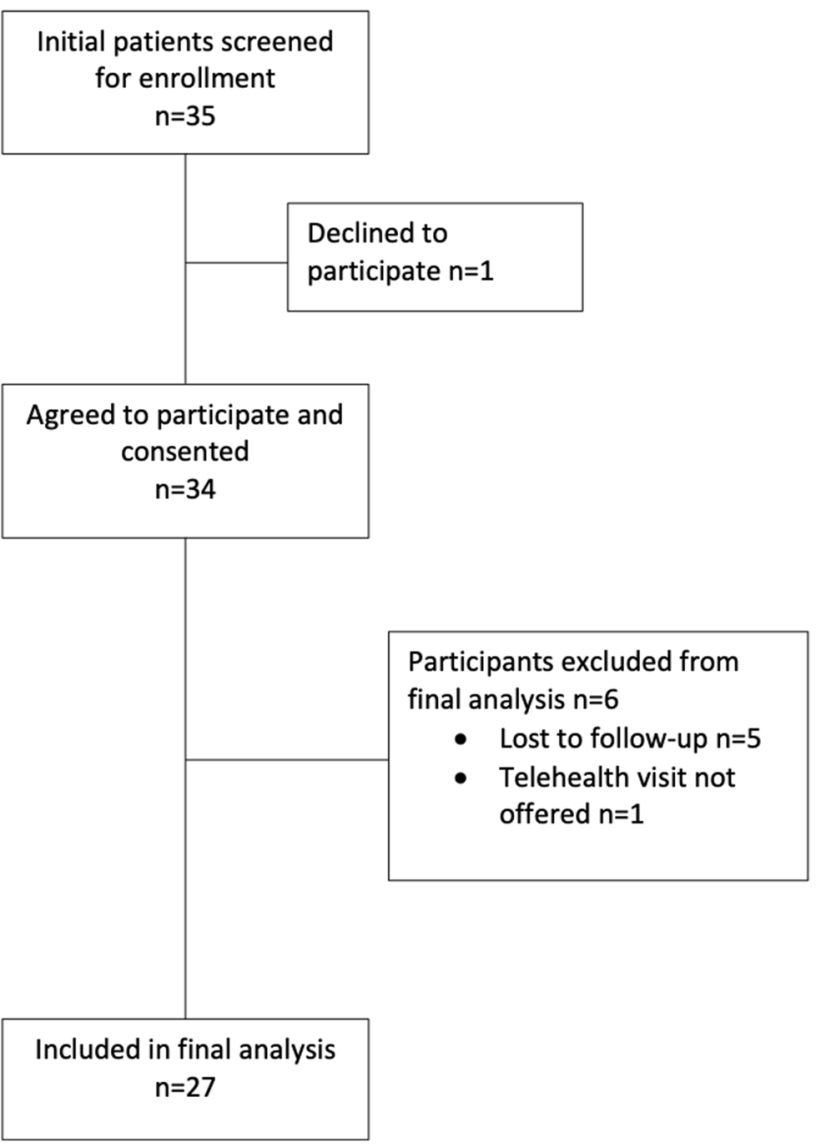

Fig. 1 Consort diagram of flow

life score was $77 \pm 19$. The mean satisfaction score for the postoperative telehealth visit was $1.2 \pm 0.3$, where a score of 1 represented 'excellent', 2 represented 'good', and 3 represented 'fair'. The majority of study participants had commercial insurance (67\%), were of non-Hispanic ethnicity (96\%), used a personal vehicle to get to their doctor's appointments (93\%), used their mobile phone for the virtual postoperative visit (52\%), and traveled between 30 and $60 \mathrm{~min}$ to get to the surgeon's office (44\%) (Table 1). Of the study participants included in the final analysis, $63 \%$ had at least 1 prior experience with telehealth. The breakdown of surgical procedures performed on the study participants included in the final analysis were as follows: 19 robotic hysterectomies (70\%), 4 robotic myomectomies (15\%), 3 robotic excisions of endometriosis (11\%), and 1 robotic adnexal surgery (4\%). There were no conversions from the planned surgical approach to an abdominal surgery. Most (85\%) of the study participants were discharged home on the same day of their surgery, with only 1 patient staying beyond postoperative day 1 .

Surgical data for the entire study sample can be found in Table 2. Of the 33 participants who underwent surgery in this study (including the 6 patients excluded from the final satisfaction analysis), 2 patients $(6.1 \%)$ received blood
Table 1 Characteristics of the sample

\begin{tabular}{ll}
\hline Patient variable & Mean \pm SD or N(\%) \\
\hline Age & $42 \pm 10$ \\
Race & \\
Caucasian & $15(56 \%)$ \\
African-American & $11(41 \%)$ \\
Other & $1(4 \%)$ \\
Ethnicity & \\
Hispanic & $1(4 \%)$ \\
Non-Hispanic & $26(96 \%)$ \\
BMI & $33 \pm 9$ \\
Insurance & \\
Commercial & $18(67 \%)$ \\
Federal & $9(33 \%)$ \\
Previous experience with video conferencing? & No $10(37 \%)$ \\
& Yes $1763 \%)$ \\
Travel time to surgeon's office & \\
$<15$ min & $5(19 \%)$ \\
15-20 min & $6(22 \%)$ \\
$30-60$ min & $12(44 \%)$ \\
$>60$ min & $4(15 \%)$ \\
Mode of transportation to surgeon's office & \\
Personal vehicle & $25(93 \%)$ \\
Ride share/Taxi & $2(7 \%)$ \\
Device used for virtual visit & \\
Computer & $13(48 \%)$ \\
Phone & $14(52 \%)$ \\
Fostoperative health-related quality of life score & $77 \pm 19$ \\
None & \\
1 area & \\
\hline & \\
&
\end{tabular}

transfusions within 30 days of their surgery. One patient was transfused on postoperative day 1 secondary to continued uterine bleeding following a robotic myomectomy, and the second patient was transfused on postoperative day 16 following an uncomplicated robotic hysterectomy. The second patient was ultimately diagnosed with unknown preoperative portal hypertension secondary to undiagnosed Wilson's disease. There were 2 postoperative readmissions, 1 of which was for the patient who received a blood transfusion on postoperative day 16 and the second of which was on postoperative day 6 for a study participant with uncontrolled pain. There were no reoperations within the study sample and no intraoperative complications.

Of the 32 postoperative telehealth visits that were initiated (including the 5 participants that were lost to followup), none were disconnected secondary to technical difficulties. All were performed using both video and audio, and only 2 participants $(6.3 \%)$ required in-person visits following 
Table 2 Surgical data for the sample

\begin{tabular}{ll}
\hline Surgical procedure & \\
Robotic hysterectomy & $20(60.6 \%)$ \\
Robotic myomectomy & $6(18.2 \%)$ \\
Robotic endometriosis excision & $5(15.2 \%)$ \\
Robotic adnexal surgery & $1(3 \%)$ \\
Diagnostic laparoscopy & $1(3 \%)$ \\
Same day discharge & $27(82 \%)$ \\
EBL & \\
$<100 \mathrm{~mL}$ & $24(73 \%)$ \\
$\geq 100 \mathrm{~mL}$ & $9(27 \%)$ \\
Conversion of surgical approach & $0(0 \%)$ \\
Postoperative complications & \\
Blood transfusion (within 30 days of surgery) & $2(6.1 \%)$ \\
Readmission & $2(6.1 \%)$ \\
Reoperation & 0 \\
Superficial Cellulitis & $1(3.0 \%)$ \\
Vaginal bleeding & $2(6.1 \%)$ \\
\hline
\end{tabular}

Estimated blood loss (EBL)

These data included all 33 participants in this study that underwent a surgical procedure

their virtual visit, both for vaginal spotting following robotic hysterectomy. One participant $(3.1 \%)$ was diagnosed with a superficial cellulitis during the virtual visit and was treated with oral antibiotics. None of the planned virtual postoperative visits had to be canceled or rescheduled for in-person visits between the date of surgery and the postoperative telehealth visit.

Postoperative telehealth visit satisfaction score was significantly associated with BMI (Pearson $r=0.45, p=0.018$ ), but not with age $(r=0.21, p=0.29)$, health-related quality of life score $(r=-0.31, p=0.11)$, race $(p=0.79)$, insurance type $(p=0.54)$, surgical procedure performed $(p=0.39)$, previous telehealth experience $(p=0.89)$, travel time to the surgeon's office $(p=0.75)$, device used for the telehealth visit $(p=0.78)$, length of stay following surgery $(p=0.60)$, estimated blood loss $(p=0.86)$, or postoperative level of functioning $(p=0.83)$.

\section{Discussion}

Our prospective data demonstrate that postoperative telehealth visits following robotic gynecologic surgery are associated with a high level of satisfaction among patients, similar to findings from other specialties $[10,11]$. The findings also support the initial hypothesis that postoperative telehealth visits are safe and feasible following major gynecologic surgery. In our study, of the 27 patients completing the post-visit survey, the average satisfaction score was just below 'excellent.' Patient satisfaction with their telehealth visit in this study was not influenced by previous telehealth experience, surgical procedure performed, overall wellbeing following surgery, travel time for office appointments, or postoperative level of functioning. We did find that BMI was significantly associated with patient satisfaction. Patients with a higher BMI were more satisfied with their postoperative telehealth visit. We do not have a rational explanation for this finding but do believe it is noteworthy and deserves further investigation.

Unfortunately, the COVID pandemic still effects how physicians practice and patients follow-up for care. Certainly, many of the adaptations implemented to accommodate social distancing during the pandemic will be longlasting, telehealth visits being one of them. Patients in an immediate postoperative state are a vulnerable patient population and every effort should be made to optimize their recovery. We believe that, at least, initial postoperative visits can be performed virtually without compromising the quality of care delivered in the appropriate clinical context while maintaining a high level of patient satisfaction.

Telehealth visits have been successfully implemented in obstetric patient populations, but to our knowledge there is no data demonstrating the safety of conducting a telehealth visit following major gynecologic surgery [6, 7]. Previous data shows that for patients undergoing pregnancy termination, virtual postprocedural visits are safe and feasible [6, 7]. Theoretical concerns over postoperative virtual visits may include a delay in diagnosis and intervention of postoperative complications, or possibly an increase in the incidence of postoperative complications. We were able to demonstrate that for the 33 surgical patients who agreed to participate in this study, it was safe to perform an initial postoperative telehealth visit except in one instance. In this instance due to a prolonged hospitalization and need for blood transfusion, the surgeon felt it was most appropriate to see this patient in person for the initial postoperative visit.

For the 27 patients included in the final analysis, there were no reported issues with connectivity for the telehealth visits. While our study was only adequately powered to assess patient satisfaction associated with postoperative virtual visits, we believe that our data support the feasibility of this type of patient encounter. Our study also found that the majority of participants traveled greater than $30 \mathrm{~min}$ to reach their surgeon's office and $7 \%$ of these participants did not have a personal vehicle to reach their surgeon's office. Telehealth visits provide an opportunity to circumvent, in part, the barriers to care our patients face in keeping scheduled appointments.

Strengths of this study primarily come from its novelty; to our knowledge it is the first of its kind. We included a variety of major gynecologic surgeries for a variety of benign indications which broadens the generalizability 
of our findings. We also had a high response rate for a survey study and are publishing on a topic that is still relevant as we continue to deliver care during the COVID-19 pandemic.

This study is not without its limitations. Primarily, this study was not adequately powered to assess the safety of postoperative virtual visits, and our small sample size must be taken into consideration when applying these findings clinically. This study also only enrolled patients from 2 minimally invasive gynecologic surgeons at a single institution, with all or the majority of participants having commercial insurance, their own means for transportation and a personal phone or computer. This highlights the relatively homogenous nature of the study population and thus limits generalizability. Furthermore, as this population lacked resource diversity, our study does not address the potential inequities regarding access to telehealth, and the impact of this approach would potentially be quite different in a diverse population with limited resources. All participants in this study underwent robotic surgery and thus we cannot know if our findings apply to patients undergoing abdominal or vaginal procedures. The study design, particularly the lack of randomization used for this investigation, is subject to selection bias with respect to patient satisfaction since all subjects agreed to participate in a telemedicine encounter. One of the study questionnaires assessing patient satisfaction with telemedicine visits was also adapted to fit the postoperative patient population and has not been previously validated. However, to our knowledge no such survey exists. Finally, for 1 of the 33 surgical patients in this study, the surgeon decided that it was more appropriate to conduct an in-person visit secondary to unforeseen postoperative complications, primarily a blood transfusion for continued uterine bleeding. This occurrence may impact our ability to comment on the safety of postoperative virtual visits, nonetheless, we believe that these visits should only be carried out based on mutual decision-making between patient and physician, and only when clinically appropriate.

Telehealth visits following minimally invasive gynecologic procedures appear to be safe and feasible, and are associated with a high level of patient satisfaction. We were able to demonstrate that patients were highly satisfied with their experience, similar to studies conducted in other surgical specialties [2,3]. Furthermore, we did not find that postoperative telehealth visits led to a delay in the diagnosis of postoperative complications or an increased demand for in-person visits. Certainly, further study with a larger sample size and randomized design is warranted to expand upon our findings.

Supplementary Information The online version contains supplementary material available at https://doi.org/10.1007/s11701-021-01354-w.
Author contributions All authors contributed to the study's conception and design. Material preparation, data collection and analysis were performed by CM, JK, EG, JG, SM, RA and GM. The first draft of the manuscript was written by JK and all authors commented on previous versions of the manuscript. All authors read and approved the final manuscript.

Funding The authors declare that no funds, grants, or other support were received during the preparation of this manuscript.

\section{Declarations}

Conflict of interest Dr. Moawad is a speaker for Intuitive Surgical. No other authors have any disclosure to report.

Ethical approval This study was approved by the local Institutional Review Board (IRB\# E-20-5088).

Consent to participate Consent was obtained from each participant in this study prior to participation.

Consent to publish Participants in this study agreed to have data from this study published.

\section{References}

1. Wosik J, Fudim M, Cameron B, Gellad ZF, Cho A, Phinney D, Curtis S, Roman M, Poon EG, Ferranti J, Katz JN, Tcheng J (2020) Telehealth transformation: COVID-19 and the rise of virtual care. J Am Med Inf Assoc 27(6):957-962. https://doi.org/ 10.1093/jamia/ocaa067.PMID:32311034;PMCID:PMC7188147

2. Walędziak M, Różańska-Walędziak A, Pędziwiatr M, Szeliga J, Proczko-Stepaniak M, Wysocki M, Stefura T, Major P (2020) Bariatric surgery during COVID-19 pandemic from patients' point of view-the results of a national survey. J Clin Med 9(6):1697. https://doi.org/10.3390/jcm9061697.PMID:32498298;PMCID: PMC7356361

3. Manz WJ, Goel R, Fakunle OP, Labib SA, Bariteau JT (2020) Feasibility of rapid development and deployment of a telemedicine program in a foot and ankle orthopedic practice. Foot Ankle Int. https://doi.org/10.1177/1071100720963059 (Epub ahead of print. PMID: 33040599)

4. Lee S, Hitt WC (2020) Clinical applications of telemedicine in gynecology and women's health. Obstet Gynecol Clin North Am 47(2):259-270. https://doi.org/10.1016/j.ogc.2020.02.002 (PMID: 32451017)

5. Mann R, van de Weijer PHM (2018) Adopting innovation in gynaecology: the introduction of e-consult. Aust N Z J Obstet Gynaecol 58(4):449-453. https://doi.org/10.1111/ajo.12764 (Epub 2018 Jan 12 PMID: 29327350)

6. Raymond E, Chong E, Winikoff B, Platais I, Mary M, Lotarevich T, Castillo PW, Kaneshiro B, Tschann M, Fontanilla T, Baldwin M, Schnyer A, Coplon L, Mathieu N, Bednarek P, Keady M, Priegue E (2019) Telabortion: evaluation of a direct to patient telemedicine abortion service in the United States. Contraception 100(3):173-177. https://doi.org/10.1016/j.contraception.2019.05. 013 (Epub 2019 Jun 4 PMID: 31170384)

7. Grossman D, Grindlay K (2017) Safety of medical abortion provided through telemedicine compared with in person. Obstet Gynecol 130(4):778-782. https://doi.org/10.1097/AOG.00000 00000002212 (PMID: 28885427) 
8. Soegaard Ballester JM, Scott MF, Owei L, Neylan C, Hanson CW, Morris JB (2018) Patient preference for time-saving telehealth postoperative visits after routine surgery in an urban setting. Surgery 163(4):672-679. https://doi.org/10.1016/j.surg.2017.08.015 (Epub 2018 Feb 3 PMID: 29398042)

9. Tofte JN, Anthony CA, Polgreen PM, Buckwalter JA, Caldwell LS, Fowler TP, Ebinger T, Hanley JM, Dowdle SB, Holte AJ, Arpey NC, Lawler EA (2020) Postoperative care via smartphone following carpal tunnel release. J Telemed Telecare 26(4):223231. https://doi.org/10.1177/1357633X18807606 (Epub 2018 Nov 14 PMID: 30428766)

10. Siow MY, Walker JT, Britt E, Kozy JP, Zanzucchi A, Girard PJ, Schwartz AK, Kent WT (2020) What was the change in telehealth usage and proportion of no-show visits for an orthopaedic trauma clinic during the COVID-19 pandemic? Clin Orthop Relat Res 478(10):2257-2263. https://doi.org/10.1097/CORR.0000000000 001396 (PMID: 32639309)

11. Grandizio LC, Mettler AW, Caselli ME, Pavis EJ (2020) Telemedicine after upper extremity surgery: a prospective study of program implementation. J Hand Surg Am 45(9):795-801. https:// doi.org/10.1016/j.jhsa.2020.06.002 (Epub 2020 Jul 18. PMID: 32693989; PMCID: PMC7368157)

12. Morgan DG, Kosteniuk J, Stewart N, O'Connell ME, Karunanayake C, Beever R (2014) The telehealth satisfaction scale: reliability, validity, and satisfaction with telehealth in a rural memory clinic population. Telemed J E Health 20(11):997-1003. https:// doi.org/10.1089/tmj.2014.0002 (Epub 2014 Oct 1. PMID: 25272141; PMCID: PMC4457516)

13. The EuroQol Group (1990) EuroQol-a new facility for the measurement of health-related quality of life. Health Policy 16(3):199-208

Publisher's Note Springer Nature remains neutral with regard to jurisdictional claims in published maps and institutional affiliations. 